\title{
Efeitos da implantação da Usina Hidrelétrica de Rosal, Rio Itabapoana, Estados do Espírito Santo e Rio de Janeiro, sobre anofelinos, planorbídeos e flebotomíneos
}

\author{
Effects of the installation of the Rosal hydroelectric power station, Itabapoana \\ River, States of Espírito Santo and Rio de Janeiro, on anophelinae, \\ planorbidae and phlebotominae
}

\author{
Helder Ricas Rezende ${ }^{1,2}$, Paulo Augusto Sessa ${ }^{2}$, Adelson Luiz Ferreira ${ }^{2}$, \\ Claudiney Biral dos Santos ${ }^{1,2}$, Gustavo Rocha Leite ${ }^{2}$ e Aloísio Falqueto ${ }^{2}$
}

\begin{abstract}
RESUMO
A construção de represas geralmente causa modificações na composição da fauna em sua área de influência. Na área de implantação da Usina Hidrelétrica de Rosal, Estados do Espírito Santo e Rio de Janeiro, foram avaliadas modificações na abundância de anofelinos, planorbídeos e flebotomíneos antes (1998-2000) e após (2000-2005) o represamento. Foram definidos nove pontos de coleta, cada qual representado por uma moradia e seus anexos, abrigos de animais domésticos e coleções hídricas num raio de 150m. Coletaram-se 103 anofelinos adultos antes do represamento e 313 depois, 200 imaturos antes e 708 depois, 868 planorbídeos antes e 486 depois, e 2.979 flebotomíneos antes e 912 depois. 0 registro de vetores dentre anofelinos, planorbídeos e flebotomíneos revela o potencial da área para transmissão de malária, esquistossomose e leishmaniose tegumentar. As transformações ambientais ocorridas, no entanto, não aumentaram o risco para ocorrência dessas doenças.
\end{abstract}

Palavras-chaves: Monitoramento de vetores. Represamento. Anofelinos. Planorbídeos. Flebotomíneos.

\section{ABSTRACT}

The construction of dams usually changes the composition of the fauna within their areas of influence. In the area where the Rosal hydroelectric power station has been installed, in the States of Espírito Santo and Rio de Janeiro, changes to the abundance of anophelinae, planorbidae and phlebotominae were evaluated before (1998-2000) and after (2000-2005) the damming. Nine sample collection points were defined, each represented by a home and its outbuildings, shelters for domestic animals and water collection points within a radius of $150 \mathrm{~m}$. One hundred and three adult anophelinae were collected before the damming and 313 afterwards; 200 immature anophelinae before and 708 afterwards; 868 planorbidae before and 486 afterwards; and 2,979 phlebotominae before and 912 afterwards. The vector record, consisting of anophelinae, planorbidae and phlebotominae, shows the potential of this area for transmission of malaria, schistosomiasis and American cutaneous leishmaniasis. However, the environmental changes did not increase the risk of the occurrence of these diseases.

Key-words: Vector monitoring. Damming. Anophelinae. Planorbidae. Phlebotominae.

A construção de represas geralmente causa modificações na composição da fauna em sua área de influência ${ }^{124}$. Estas modificações têm direcionado pesquisas para os vetores de doenças endêmicas, visto que podem favorecer a transmissão em áreas de baixa endemicidade ou propiciar a instalação de novos focos de doenças ${ }^{4624}$.

Dentre os invertebrados vetores de doenças, os anofelinos e os planorbídeos são os que parecem sofrer maior influência destes empreendimentos ${ }^{123}$. 0 vetor principal da malária nas Américas, Anopheles darlingi Root, 1926, tem preferência por

1. Núcleo de Entomologia, Secretaria Estadual de Saúde do Espírito Santo, Vitória, ES. 2. Unidade de Medicina Tropical, Universidade Federal do Espírito Santo, Vitória, ES. Endereço para correspondência: MSc. Helder Ricas Rezende. Dept ${ }^{\underline{0}}$ de Patologia/ UFES. Av Marechal Campos 1468, 29040-090 Vitória, ES.

Tel: 5527 3335-7294; Fax: 5527 3324-2038

e-mail: heldericas@hotmail.com

Recebido para publicação em 02/09/2008

Aceito em 20/03/2009 criadouros representados por grandes coleções de água doce ${ }^{323}$. Os planorbídeos, vetores da esquistossomose, têm sido associados aos ambientes modificados por projetos como a construção de usinas hidrelétricas e represas ${ }^{126}$. A presença de um volume grande de água também causa modificações em grupos não relacionados diretamente com o ambiente aquático, como é o caso dos flebotomíneos ${ }^{4}$.

Considerando a implantação da Usina Hidrelétrica de Rosal (UHER) na divisa dos Estados do Espírito Santo e Rio de Janeiro, avaliou-se o impacto das modificações ambientais sobre anofelinos, planorbídeos e flebotomíneos e as implicações na epidemiologia da malária, esquistossomose e leishmaniose.

\section{MATERIAL E MÉTODOS}

Área de estudo. A Usina Hidrelétrica de Rosal foi construída no Rio Itabapoana, na divisa entre os municípios de Guaçuí e 
São José do Calçado, no Estado do Espírito Santo, e o município de Bom Jesus do Itabapoana, no Estado do Rio de Janeiro, em uma altitude de $600 \mathrm{~m}$ (Figura 1). 0 reservatório possui extensão de 6km e área de inundação de 128,5ha, com volume acumulado de $10,9 \times 10^{6} \mathrm{~m}^{3}$. Primitivamente a região era coberta por floresta Atlântica estacional semidecidual, da qual restaram apenas fragmentos pequenos principalmente nas partes altas dos morros $^{216}$. 0 clima na região é ameno e o relevo acidentado. A ocorrência maior de chuvas é durante os meses de novembro a fevereiro, e o período mais seco estende-se de julho a setembro. A temperatura média nos meses mais quentes aproxima-se de $24^{\circ} \mathrm{C}$, e nos meses mais frios é em torno de $18^{\circ} \mathrm{C}$. A umidade relativa mantém-se próxima de $80 \%$ durante todo 0 ano ${ }^{2915}$.

Coleta e identificação. As amostragens, na fase de construção (FC), ocorreram entre os anos de 1998 e 2000, totalizando cinco campanhas com intervalos médios de cinco meses. Na fase de operação (FO), as amostragens ocorreram entre os anos 2000 e 2005, totalizando 10 campanhas, também com intervalos médios de cinco meses. Tanto na FC quanto na FO, as amostragens contemplaram todas as estações do ano, englobando períodos secos e chuvosos em números semelhantes.

As coletas foram feitas simultaneamente em três pontos por dia, durante três dias consecutivos, compreendendo nove pontos por campanha. Cada ponto de coleta abrangia uma moradia e seus anexos, além de abrigos de animais domésticos e coleções hídricas em um raio de $150 \mathrm{~m}$. As coletas de anofelinos e flebotomíneos adultos ocorreram durante as quatro horas após o crepúsculo, por meio de busca ativa com capturador de Castro e passiva com armadilha luminosa CDC (Centers for Disease Control and Prevention $)^{22}$ instalada no peridomicílio. Na busca ativa, os insetos foram coletados em paredes internas e externas das residências, anexos domiciliares, abrigos de animais e troncos de árvores. Os imaturos de anofelinos e os planorbídeos foram coletados por varredura, durante quatro horas, no período diurno, em remansos de cursos naturais de água, alagados, brejos e açudes. Os imaturos de anofelinos foram coletados com redes pesca-larvas, pipetas e bacias e os planorbídeos com conchas metálicas ${ }^{10}$.

A identificação dos anofelinos baseou-se na chave de Consoli \& Lourenço-de-Oliveira ${ }^{3}$ e a dos flebotomíneos baseou-se em Galati ${ }^{11}$. Dez por cento dos planorbídeos foram identificados com base nos caracteres anatômicos do sistema renal-genital e o restante com base na morfologia das conchas. Estes foram esmagados entre placas de Petri e examinados em microscópio estereoscópico para verificação da infecção por Schistosoma mansoni Sambon, $1907^{10}$.

Análise. As diferenças na abundância relativa média entre os grupos e entre as espécies na FC e FO foram avaliadas pelo teste estatístico não-paramétrico de Wilcoxon para amostras relacionadas, pelo método exato. A aplicação do teste de Wilcoxon foi precedida pela avaliação da normalidade dos dados por meio da análise de Kolmogorov-Smirnov ${ }^{29}$.

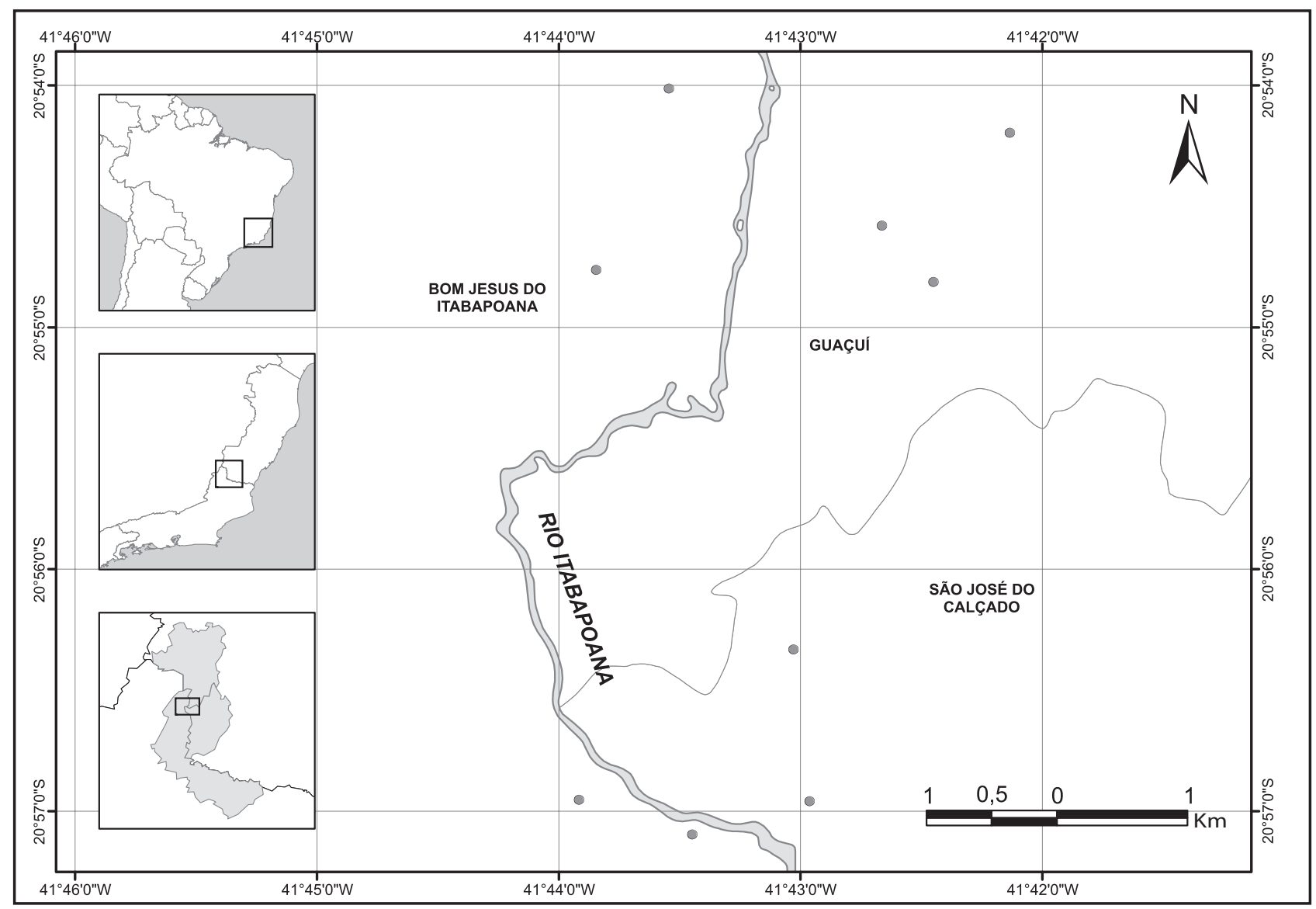

FIGURA 1

Localização geográfica da área de estudo e pontos amostrais no Rio Itabapoana, onde foi implantada a Usina Hidrelétrica de Rosal, abrangendo municípios dos Estados do Espírito Santo e Rio de Janeiro. 


\section{RESULTADOS}

Anofelinos. Entre adultos e imaturos foram obtidos 1.324 exemplares agrupados em nove espécies. $\mathrm{Na} \mathrm{FC}$, foram coletados 103 adultos e 200 imaturos, e na FO foram 313 adultos e 708 imaturos. Considerando-se o total de exemplares adultos e imaturos coletados nas duas fases, as espécies mais frequientes foram Anopheles evansae (Brethes, 1926) (30,3\%), Anopheles strodei Root, 1926 (28,6\%) e Anopheles argyritarsis RobineauDesviody, 1827 (28,5\%), seguidos de Anopheles albitarsis Lynch-Arribálzaga, 1878 (6,1\%) e Anopheles lutzi Cruz, 1901 (5,1\%). As demais espécies, Anopheles galvaoi Causey, Deane \& Deane, 1943, Anopheles oswaldoi (Peryassu, 1922), Anopheles triannulatus (Neiva \& Pinto, 1822) eAnopheles minor Lima, 1929, corresponderam a 1,3\% do total e ocorreram apenas na FO.

0 teste de Wilcoxon revelou que a abundância de anofelinos não variou significativamente após a formação do lago da UHER. Considerando as espécies separadamente, aumentou a abundância de adultos de Anopheles albitarsis $(Z=-2,38$ e $p=0,01)$ e de seus imaturos $(Z=-2,11$ e $p=0,02)$, bem como a de imaturos de Anopheles evansae $(Z=-1,84$ e $p=0,04)$ eAnopheles strode $i$ $(Z=-2,19$ e $p=0,01)$. As demais espécies não variaram (Figura 2).

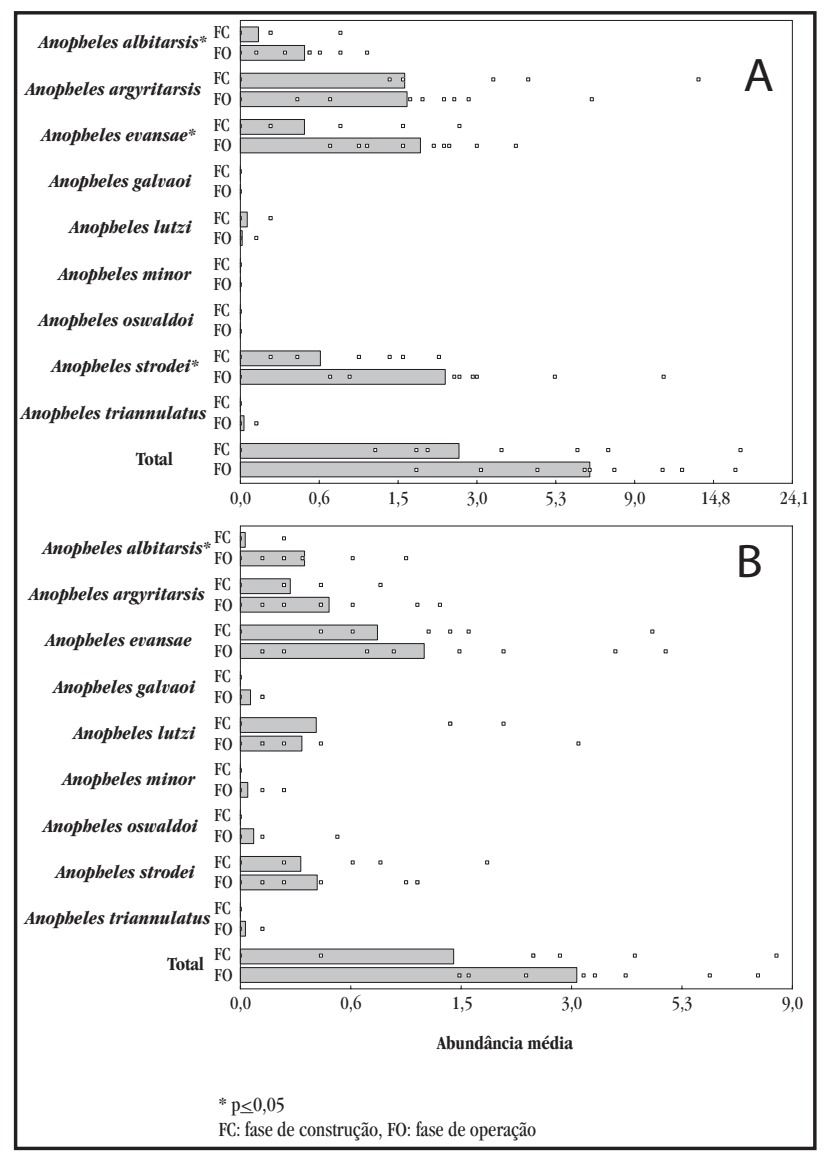

FIGURA 2

Abundância média dos imaturos (A) e adultos (B) de cada espécie de anofelino e de toda a população por ponto de coleta, nas fases de construção, entre 1998 e 2000 e operação, entre 2000 e 2005, da Usina Hidrelétrica de Rosal, no Rio Itabapoana, em municípios dos Estados do Espírito Santo e Rio de Janeiro. Cada quadrado representa um ponto de coleta e a barra representa a média. 0 eixo $\mathrm{X}$ está na escala logarítmica, mas com os valores reais.
Planorbídeos. Dentre os 1.354 planorbídeos, foramidentificados 1.305 exemplares de Biomphalaria tenagophila (Orbigny, 1835), dos quais 820 foram coletados na FC e 485 na FO. Biomphalaria glabrata (Say, 1818) contribuiu com 49 indivíduos, sendo 48 coletados na FC e apenas um na FO. Nenhum dos 1.175 exemplares examinados de Biomphalaria tenagophila e dos 44 de Biomphalaria glabrata estava infectado por Schistosoma mansoni.

A análise revelou redução da abundância de Biomphalaria tenagophila $(Z=-2,55$ е $p=0,01)$ bem como de Biomphalaria glabrata $(Z=-2,38$ e $p=0,02)$, após o represamento.

Flebotomíneos. Foram coletados 3.891 exemplares agrupados em oito espécies. Nissomyia intermedia (Lutz \& Neiva, 1912) foi mais frequiente, com 2.585 indivíduos coletados na FC e 721 na FO, seguida por Nissomyia whitmani (Antunes \& Coutinho, 1939) que apresentou 354 exemplares na FC e 162 na FO. As demais espécies, Evandromyialenti (Mangabeira, 1938), Micropygomyia quinquefer (Dyar, 1929), Pintomyia fischeri (Pinto, 1926), Migonemyiamigonei, Lutzomyia amarali (Barreto \& Coutinho, 1940) e Brumptomyia avellari (Costa Lima, 1932) somaram 69 exemplares nas duas fases. Lutzomyia amarali só foi coletada naFC, enquanto Pintomyia fischeri, Migonemyiamigonei (França, 1920) eBrumptomyia avellari (Costa Lima, 1932) só ocorreram na FO.

Houve redução da abundância de flebotomíneos depois da formação do lago $(Z=-1,95$ e $p=0,03)$. Considerando as espécies separadamente, somente para Nissomyia intermedia a redução foi significativa $(Z=-1,95$ e $p=0,03)$ (Figura 3).

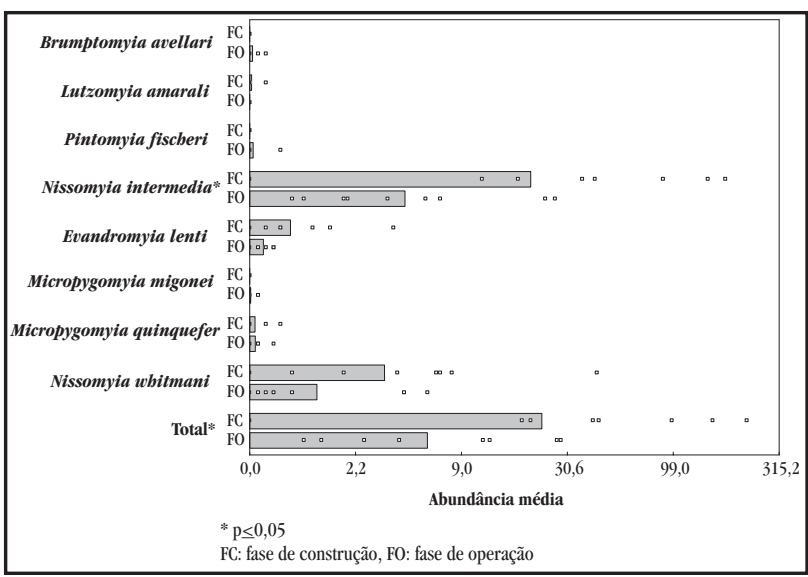

FIGURA 3

Abundância média de cada espécie de flebotomíneo e de toda a população por ponto de coleta, nas fases de construção, entre 1998 e 2000, e operação, entre 2000 e 2005 , da Usina Hidrelétrica de Rosal, no Rio Itabapoana, em municípios dos Estados do Espírito Santo e Rio de Janeiro. Cada quadrado representa um ponto de coleta e a barra representa a média. 0 eixo $\mathrm{X}$ está na escala logarítmica, mas com os valores reais.

\section{DISCUSSÃO}

0 impacto mais significativo durante a FC foi o aumento de residentes temporários devido a afluxo de cerca de quatrocentos trabalhadores. Este aumento populacional implicou risco de introdução de agentes infecciosos, além de proporcionar maior perturbação no ambiente, produção de lixo e eliminação de dejetos. Na FO o maior impacto foi a formação do lago, bem como a saída de moradores e trabalhadores da área. 
Embora a abundância de anofelinos não tenha aumentado após o represamento, é possível que a maior oferta e qualidade de criadouros justifiquem o aumento da abundância de Anopheles albitarsis, Anopheles strodei e Anopheles evansae, bem como o maior número de espécies coletadas na FO.

Dentre as espécies de anofelinos coletadas, destaca-se Anopheles albitarsis, considerado o vetor principal de malária em alguns estudos ${ }^{51}$. Na maioria das vezes, entretanto, lhe foi atribuído apenas papel secundário em áreas onde a transmissão é mantida por Anopheles darlingi. As espécies Anopheles strodei, Anopheles evansae, Anopheles galvaoi, Anopheles oswaldoi e Anopheles triannulatus também são consideradas vetores secundários em áreas onde Anopheles darlingi é o vetor principal. Já Anopheles argyritarsis, Anopheles minor e Anopheles lutzi não são apontadas como transmissoras da malária humana ${ }^{323}$.

O número reduzido de exemplares de Anopheles albitarsis e a ausência de outras espécies de importância na epidemiologia da malária na área da UHER indicam que são limitadas as possibilidades de instalação e propagação dos plasmódios humanos. Reforça esta idéia o estudo realizado no Estado do Espírito Santo, que associa a distribuição da malária à presença de Anopheles darlingi e Anopheles aquasalis (Curry, 1932), que não são encontradas em áreas com temperatura amena e relevo acidentado, como se observa na área da $\mathrm{UHER}^{18}$. De fato nenhum caso autóctone da doença foi registrado até o momento na área (Secretaria de Estado da Saúde do Espírito Santo: dados não publicados).

Biomphalaria tenagophila e Biomphalaria glabrata já haviam sido registradas no Rio Itabapoana ${ }^{19}$. As condições climáticas locais parecem mais propícias à ocorrência de Biomphalaria tenagophila, tendo influenciado seu predomínio sobre Biomphalaria glabrata, que é o vetor principal da esquistossomose no Brasil ${ }^{28}$. Na área da UHER, Biomphalaria tenagophila foi encontrada em diversos pontos de coleta, no entanto foi mais numerosa em dois córregos que, em virtude de obras da usina, apresentaram a velocidade do fluxo reduzida na FC. A alteração do fluxo de água de lótico para lêntico e o acúmulo maior de matéria orgânica lançada nos córregos pelos trabalhadores da usina facilitaram a proliferação dos planorbídeos. Na FO, a maioria dos trabalhadores deixou a área e a velocidade do fluxo de água dos dois córregos aumentou novamente, justificando em parte a redução na abundância de planorbídeos.

No município de Porciúncula, situado na bacia do Rio Itabapoana, no Estado do Rio de Janeiro, foram relatados 37 casos de esquistossomose ${ }^{25}$. Portanto, a presença de Biomphalaria tenagophila e Biomphalaria glabrata na área de estudo permite inferir a possibilidade de ocorrência de esquistossomose, mesmo que a doença não seja endêmica na região e que os moluscos examinados estivessem negativos para Schistosoma mansoni ${ }^{19}$. Ressalta-se ainda o risco de contaminação do rio devido à poluição por fezes humanas provenientes da Vila de Rosal e arredores. Na triagem pré-admissional, para as obras da UHER, foram identificados 13 trabalhadores oriundos de área endêmica, infectados por Schistosoma mansoni ${ }^{2}$.
Quanto aos flebotomíneos, é possível que a maior abundância na FC tenha sido favorecida pela disponibilidade de alimento para as fêmeas e de matéria orgânica, que permitia a formação de criadouros. A saída dos trabalhadores e de moradores e o alagamento de nichos nas grotas e fundo dos vales parecem ter provocado a inversão deste cenário na FO, promovendo a redução na abundância de flebotomíneos.

Considerando o predomínio e o número elevado de exemplares de Nissomyia intermedia coletados durante o monitoramento, atenção especial deve ser dada a este flebotomíneo, que apresenta papel relevante na transmissão domiciliar de Leishmania braziliensis Vianna, 1911 em ambientes alterados ${ }^{7} 81217$. Nissomyia whitmani também é importante na epidemiologia da leishmaniose tegumentar, tanto em áreas florestais como em ambientes modificados, promovendo o aporte de leishmânias de reservatórios silvestres para os animais domésticos no peridomicílio ${ }^{72}{ }^{17}$. A pequena abundância de Migonemyia migonei indica que a espécie apresenta pouca importância epidemiológica na área da UHER. As demais espécies, Evandromyia lenti, Micropygomyia quinquefer, Pintomyia fischeri, Lutzomyia amarali e Brumptomyia avellari não têm sido citadas como vetores de leishmaniose ${ }^{12}$.

Mesmo com a redução na abundância de Nissomyia intermedia depois do represamento, a área de influência da UHER não está livre da ocorrência da leishmaniose tegumentar, uma vez que foram notificados 27 casos dessa doença nos municípios de Guaçuí e São José do Calçado, entre 1990 e 2002 (Secretaria de Estado da Saúde do Espírito Santo: dados não publicados).

A maior disponibilidade e oferta permanente de criadouros, variações no nível do lago, presença de vegetação nativa ou de reflorestamento nas suas margens e o acúmulo de sedimento no ambiente lêntico do lago, estão entre as transformações ambientais provocadas pela implantação de usinas hidrelétricas relacionadas a modificações na fauna de vetores ${ }^{4132023242627}$. 0 fluxo migratório com introdução de patógenos e o aumento populacional ou introdução de vetores representam maior risco de transmissão de doenças ${ }^{61314}$. Em nosso estudo, a abundância da população de anofelinos não cresceu significativamente, visto que o aumento da disponibilidade de criadouros foi acompanhado pela redução na quantidade de alimento disponível para as fêmeas. Os pequenos remanescentes de floresta Atlântica secundária parecem abrigar poucos animais, ficando parte da população de anofelinos mantida na dependência de sangue humano e de animais domésticos. A população de planorbídeos parece ter aumentado durante a FC, em função de alterações ambientais temporárias, revertidas posteriormente, o que teria determinado a redução dessa população na FO. Para os flebotomíneos, além da diminuição da oferta de sangue para as fêmeas, houve redução de criadouros, diminuindo os riscos de transmissão nos pontos analisados.

0 registro de vetores entre a fauna de anofelinos, planorbídeos e flebotomíneos na área de estudo revela seu potencial para transmissão de malária, esquistossomose e leishmaniose tegumentar. As alterações provocadas pela formação do lago, de porte relativamente pequeno, e pelo fluxo de pessoas, não foram suficientes para aumentar significativamente o risco de ocorrência dessas endemias. 


\section{AGRADECIMENTOS}

A Márcia Visibelli, José Daniel Simões e demais funcionários da Rede Paranapanema/Central Elétrica de Minas Gerais. Aos companheiros do Núcleo de Entomologia da Secretaria Estadual de Saúde: Agenor Oliveira, Edmar Thomaz, Izaias Albino, João Batista, Jones Oliveira e Nely Caldeira, fundamentais na execução deste trabalho.

\section{REFERÊNCIAS}

1. Camargo S. Impacto do desenvolvimento na expansão da esquistossomose. Revista do Instituto de Medicina Tropical de São Paulo 22:117-119, 1980.

2. Companhia de Eletricidade do Rio de Janeiro. Relatório de Impacto Ambiental. Aproveitamento Hidrelétrico do Rio Itabapoana, Usina Hidrelétrica de Rosal, Rio de Janeiro/Espírito Santo ( ${ }^{\circ}$ 504.03), 1992.

3. Consoli RAGB, Lourenço-de-Oliveira R. Principais mosquitos de importância sanitária no Brasil. Editora Fundação Oswaldo Cruz, Rio de Janeiro, 1994.

4. Consolim J, Luz E, Torres PB. Flebótomos (Diptera; Psychodidae) da área do reservatório da usina hidrelétrica de Itaipu, Estado do Paraná, Brasil. Cadernos de Saúde Pública 6:86-89, 1990.

5. Coutinho JO. Distribuição geográfica dos anofelinos do Estado do Rio de Janeiro. Arquivos de Higiene e Saúde Pública 11:439-457, 1946.

6. Falavigna-Guilherme AL, Silva AM, Guilherme EV, Morais DL. Estudo retrospectivo da prevalência de malária e do gênero Anopheles na área de influência do reservatório de Itaipu Binacional. Revista do Instituto de Medicina Tropical de São Paulo 47:81-86, 2005

7. Falqueto A. Especificidade alimentar de flebotomíneos em duas áreas endêmicas de leishmaniose tegumentar no Estado do Espírito Santo. Tese de Doutorado, Fundação Oswaldo Cruz, Rio de Janeiro, 1995.

8. Falqueto A, Sessa PA, Ferreira AL, Vieira VP, Santos CB, Varejão JBM, Cupolillo E, Porrozzi R, Carvalho-Paes LE, Grimaldi Jr G. Epidemiological and clinical features of Leishmania (Viannia) braziliensis American cutaneous and mucocutaneous leishmaniasis in the State of Espírito Santo, Brasil. Memórias do Instituto Oswaldo Cruz 98:1003-1010, 2003.

9. Feitoza LR. Carta Agroclimática do Espírito Santo. Governo do Estado do Espírito Santo: Secretaria de Estado da Agricultura \& Empresa Capixaba de Pesquisa Agropecuária, 1986.

10. Fundação Nacional de Saúde. Controle da esquistossomose. Operações de malacologia/Diretrizes técnicas, Ministério da Saúde, 1995.

11. Galati EAB. Morfologia e taxonomia. Morfologia, terminologia de adultos e identificação dos táxons da América. In: Rangel EF, Lainson R (eds) Flebotomíneos do Brasil, $1^{a}$ edição, Editora da Fundação Oswaldo Cruz, Rio de Janeiro, p.53-175, 2003

12. Grimaldi Jr G, Tesh RB, McMahon-Pratt D. A review of geographical distribution and epidemiology of leishmaniasis in the New World. The American Journal of Tropical Medicine and Hygiene 41:687-725, 1989.

13. Guimarães AE, Gentile C, Alencar J, Lopes CM, Melo RP. Ecology of Anophelinae (Diptera, Culicidae), malaria vectors around the Serra da Mesa reservoir, State of Goiás, Brazil. 1 - Frequency and climatic factors. Cadernos de Saúde Pública 20:291-302, 2004

14. Guimarães AE, Melo RP, Lopes CM, Alencar J, Gentile C. Prevalência de anofelinos (Diptera: Culicidae) no crepúsculo vespertino em área da usina hidrelétrica de Itaipu, no Município de Guaíra, Estado do Paraná, Brasil. Memórias do Instituto Oswaldo Cruz 92:745-754, 1997.

15. Hijmans RJ, Cameron SE, Parra JL, Jones PG, Jarvis A. Very high resolution interpolated climate surfaces for global land areas. International Journal of Climatology 25:1965-1978, 2005.

16. Instituto Nacional de Pesquisas Espaciais, Fundação SOS Mata Atlântica. Atlas dos remanescentes florestais de Mata Atlântica - Período: 1995-2000. Fundação SOS Mata Atlântica, São Paulo, 2002.

17. Lainson R. The american leishmaniasis: some observations on their ecology and epidemiology. Transactions of the Royal Society of Tropical Medicine and Hygiene 77:569-596, 1983

18. Meneguzzi VC. Importância vetorial das espécies de anofelinos (Diptera: Culicidae) na transmissão de malária e estudo dos fatores geoclimáticos determinantes de sua distribuição geográfica no Estado do Espírito Santo. Dissertação de Mestrado, Universidade Federal do Espírito Santo, Vitória, 2006.

19. Paraense WL, Alencar JTA, Corrêa LR. Distribuição dos planorbídeos e prevalência da xistosomose mansoni no Estado do Espírito Santo. Memórias do Instituto Oswaldo Cruz 78:373-384, 1983.

20. Paula MB, Gomes AC. Culicidae (Diptera) em área sob influência de construção de represa no Estado de São Paulo. Revista de Saúde Pública 41:284-289, 2007.

21. Silva-Vasconcelos A, Kató MYN, Mourão EN, Souza RTL, Lacerda RNL, Sibajev A, Tsouris P, Póvoa MM, Momen H, Rosa-Freitas MG. Biting indices, host-seeking activity and natural infection rates of anopheline species in Boa Vista, Roraima, Brazil from 1996 to 1998. Memórias do Instituto Oswaldo Cruz 97:151-161, 2002

22. Sudia WD, Chamberlain RW. Battery operated light trap, an improved model. Mosquito News 22:126-129, 1962.

23. Tadei WP, Thatcher BD, Santos JMM, Scarpassa VM, Rodrigues IB, Rafael MS Ecology observations on anophelinae vectors of malaria in the brazilian amazon. American Journal of Tropical Medicine and Hygiene 59:325-335, 1998.

24. Teodoro U, Guilherme ALF, Lozovei AL, Salvia-Filho VL, Fukushigue Y, Spinosa RP, Ferreira MEMC, Barbosa OC, Lima EM. Culicídeos do lago de Itaipu, no Rio Paraná, Sul do Brasil. Revista de Saúde Pública 29:6-14, 1995.

25. Thiengo SC, Mattos AC, Santos SB, Fernandez MA. Freshwater snails and schistosomiasis mansoni in the state of Rio de Janeiro, Brazil: VI - Noroeste Fluminense Mesoregion. Memórias do Instituto Oswaldo Cruz 101:239-245, 2006

26. Thiengo SC, Santos SB, Fernandez MA. Malacofauna límnica da área de influência do lago da usina hidrelétrica de Serra da Mesa, Goiás, Brasil. I. Estudo qualitativo. Revista Brasileira de Zoologia 22:867-874, 2005.

27. Tubaki RM, Menezes RMT, Cardoso Jr RP, Bergo ES. Studies on entomological monitoring: mosquito species frequency in riverine habitats of the Igarapava dam, southern region, Brazil. Revista do Instituto de Medicina Tropical de São Paulo 46:223-229, 2004

28. Valadão R, Andrade RM. Interações de planorbídeos vetores da esquistossomose mansoni e o problema da expansão da endemia na região amazônica. Revista de Saúde Pública 25:353-358, 1991.

29. Zar JH. Biostatistical Analysis. Prentice Hall, Upper Saddle River, 1999. 